\title{
SEGURIDAD ALIMENTARIA FAMILIAR Y HOGARES SUSTENTABLES DE ALUMNOS PRACTICANTES DE NUTRICIÓN DURANTE LA PANDEMIA DE COVID 19.
}

\author{
FAMILY FOOD SECURITY AND SUSTAINABLE HOUSEHOLDS OF STUDENT NUTRITION PRACTITIONERS \\ DURING THE COVID 19 PANDEMIC. \\ Crocker-Sagastume René Cristóbal*, Esparza Alvarado Nohemí Rahel*, Huerta García Lourdes Concepción*, \\ Pérez Boarín Ariadna Lizbeth*. \\ * Universidad de Guadalajara, México.
}

\section{RESUMEN}

Introducción. Se analiza la situación de seguridad y vulnerabilidad alimentaria familiar de alumnos practicantes de Nutrición. Objetivo: Mejorar las formas de respuesta educativas vinculadas a hogares sustentables en un Centro de la Universidad de Guadalajara, en el contexto de la pandemia de COVID 19. Material y Método: Se seleccionan a 67 familias de un universo de 122 familias de alumnos practicantes. Se aplica un diseño metodológico de investigación acción participativa; se recolectan datos con encuesta, que incluye elementos de la cadena epidemiológica de seguridad alimentaria y el análisis del discurso escrito para evaluar las respuestas de alumnos a la problemática familiar. Resultados: El 70\% obtiene alimentos del mercado y supermercado, $10.4 \%$ de producción en casa; la mitad de la población consume diariamente lácteos, cárnicos y azúcares. La prevalencia de sobrepeso/obesidad es de $46.25 \%$; el $6 \%$ ha padecido Covid-19 y $10.5 \%$ presenta alguna enfermedad previa. El $91.84 \%$ de los alumnos realiza intervención en aprovechamiento y producción de alimentos para mejorar su situación de vulnerabilidad alimentaria. Conclusiones: Las familias de los alumnos tienen inseguridad y vulnerabilidad alimentaria de acuerdo con indicadores de producción sustentable y consumo de alimentos, esto impacta en el estado nutricio y prevalencia de enfermedad crónico-degenerativas asociadas a COVID-19.

Palabras Clave: Seguridad alimentaria, hogares sustentables, COVID 19.

\section{ABSTRACT}

Introduction: The family food security and vulnerability situation of students practicing Nutrition is analyzed. Objective: To improve the forms of educational response linked to sustainable homes in a Center of the University of Guadalajara, in the context of the COVID 19 pandemic. Material and method: 67 families are selected from a universe of 122 families of practicing students. A participatory action research methodological design is applied; Data are collected with a survey, which includes elements of the epidemiological chain of food safety and the analysis of written discourse to evaluate the responses of students to family problems. Results: $70 \%$ obtain food from the market and supermarket, $10.4 \%$ from home production; half of the population consumes dairy, meat, and sugars daily. The prevalence of overweight / obesity is $46.25 \%$; $6 \%$ have suffered from Covid-19 and $10.5 \%$ have a previous illness. $91.84 \%$ of the students perform intervention in the use and production of food to improve their situation of food vulnerability. Conclusions: The families of the students have food insecurity and vulnerability according to indicators of sustainable production and food consumption, this impacts on the nutritional status and prevalence of chronic-degenerative diseases associated with COVID-19.

Key words: Food security, sustainable households, COVID 19.

Correspondencia: René Cristóbal Crocker Sagastume rene.crocker@academicos.udg.mx

Recibido: 31 de marzo 2021, aceptado: 09 de agosto 2021

(c) Autor2021

(c) (1)

DOI: https://doi.org/10.29105/respyn20.4-1

Citation: Crocker-Sagastume R.C., Esparza Alvarado N.R., Huerta García L.C., Pérez Boarín A.L. (2021)

Seguridad alimentaria familiar y hogares sustentables de alumnos practicantes de nutrición durante la pandemia de COVID 19. Revista Salud Pública y Nutrición, 20 (4), 1-10. 


\section{Artículo Original}

\section{Introducción}

En la presente investigación se analiza la situación de seguridad y vulnerabilidad alimentaria de las familias de los alumnos practicantes de la Carrera de Nutrición del Centro Universitario de Ciencias de la Salud de la Universidad de Guadalajara con el objetivo de mejorar las formas de respuesta socioeducativas vinculadas a hogares sustentables en el contexto de la pandemia de COVID 19 y la crisis socio-ambiental que se vive en el planeta.

A partir de la pandemia de COVID 19 generada por cambios socio ambientales que afectan el equilibrio de la naturaleza, entre otras causas, se ha profundizado la necesidad de que las escuelas y facultades que forman profesionales de la Nutrición, analicen la situación de seguridad alimentaria de las familias de sus alumnos, para generar acciones que incidan en el desarrollo de hogares sustentables que permitan una nueva cultura de cuidado del planeta, a partir de aportar soluciones desde sus propios entornos ambientales socio-familiares.

Para abordar el problema de la seguridad y vulnerabilidad alimentaria familiar de los alumnos practicantes y las formas de respuesta de la institución educativa a sus espacios de confinamiento durante la pandemia de COVID 19, se formulan dos preguntas de investigación: la primera, ¿Cuál es la situación de salud alimentaria de las familias de los alumnos practicantes de nutrición durante la pandemia por COVID 19, valorada a través del análisis de su situación de seguridad y vulnerabilidad alimentaria durante el otoño de 2020? La segunda, ¿Cuáles son las acciones prioritarias para establecer un programa participativo de hogares sustentables para prevenir la problemática de salud alimentaria familiar en el contexto de la pandemia de COVID 19 y la problemática socio-ambiental?

La pandemia por COVID-19 representa un importante riesgo de adquirir o bien incrementar el grado de inseguridad alimentaria en los hogares. Las medidas empleadas para controlar y evitar la propagación del virus han representado un importante impacto en la economía, lo cual, ha repercutido negativamente en muchos hogares. El aumento en los precios de los alimentos, el gasto en alimentos de los hogares y las restricciones económicas han sido reconocidos como factores causales de hasta el $50 \%$ de la inseguridad alimentaria en hogares de estudiantes universitarios (Eche, et al, 2018).

$\mathrm{Al}$ analizar los antecedentes de estudios que vinculan la seguridad alimentaria y los factores asociados con COVID 19 relacionados con hogares sustentables, se encuentra que en países como Jordania, las medidas de aislamiento representaron un incremento en la inseguridad alimentaria del $23,1 \%$, donde aspectos como el ingreso mensual per cápita y el número de miembros de la familia se asocia principalmente con la inseguridad alimentaria moderada y severa, mientras que el rango de edad entre los 18 y 30 años, así como vivir en propiedades alquiladas también incrementa la inseguridad alimentaria severa (Elsahoryi, et al, 2020).

En México se reconoce un porcentaje promedio de inseguridad alimentaria de $69.5 \%$, cuando se analizan las categorías de disponibilidad y consumo de alimentos. Las personas de escasos recursos son la población con mayor riesgo de encontrarse en una situación de inseguridad alimentaria, representando un porcentaje de $85.8 \%$, seguido de aquellas personas que viven en zonas rurales con un porcentaje de inseguridad alimentaria del $78 \%$ (Mundo-Rosas, et al, 2018).

Al revisar antecedentes de la asociación entre comorbilidades vinculadas a la nutrición y COVID 19, los estudios muestran significancia estadística. De acuerdo a Posso y colaboradores (2020) en España se encontró que la prevalencia de al menos una comorbilidad en pacientes positivos a COVID19 fue de un $81.9 \%$, donde la hipertensión fue la más frecuente con un porcentaje de $64.6 \%$, seguida por enfermedad renal crónica, diabetes y enfermedad respiratoria crónica.

En México, entre los factores de letalidad en pacientes con COVID-19 se encuentran algunas comorbilidades como diabetes de aparición temprana, obesidad, enfermedad pulmonar obstructiva crónica, edad avanzada, hipertensión, inmunosupresión y enfermedad renal crónica. El efecto de la diabetes sobre la letalidad por COVID19 está relacionada en un $49.5 \%$ por la presencia de obesidad (Bello-Chavolla, et al, 2020).

También se reconoce que la presencia de tres o más comorbilidades en pacientes positivos a COVID-19 


\section{Artículo Original}

presenta mayor tasa de letalidad, riesgo de hospitalización, neumonía e ingreso a unidad de cuidados intensivos (Kammar-García, et al, 2020).

La importancia del estudio está relacionada con el análisis de las características que conforman un hogar sustentable dentro de las familias de los alumnos, lo que permite acercarse a la situación de seguridad alimentaria durante la contingencia por COVID-19. Es una herramienta para llevar a cabo el diagnóstico y realizar propuestas de intervención que permitan fomentar la implementación de hogares sustentables en las familias de los alumnos, a través del cambio de modos de vida, aprovechando la situación de aislamiento durante la cual permanecen en sus hogares.

La implementación de estrategias de acción participativa, promovidas por alumnos practicantes de nutrición en sus propios hogares, tiene la finalidad de mejorar la seguridad alimentaria y busca transformar con ayuda de la colaboración familiar hogares sustentables que permitan alcanzar objetivos nutricionales y la sostenibilidad socio-ambiental urbana. Los objetivos de las estrategias de acción participativa abordadas en el presente estudio, tienen la finalidad de reducir el impacto socio-ambiental urbano, mediante mejoras del uso de agua y tierra, el aprovechamiento del suelo y el espacio; dietas sostenibles para la seguridad alimentaria y nutrición, las cuales, se caracterizan por ser dietas basadas en plantas, la reducción en el consumo de carne y aumento en el consumo de pescado. También involucran disminuir la cantidad de productos lácteos, como bocadillos, pan blanco y bebidas azucaradas (Wilson, et al, 2019).

Como fundamento teórico-conceptual del estudio se recuperan y construyen las siguientes definiciones: seguridad alimentaria, inseguridad alimentaria, dietas sostenibles, modos de vida sustentables, hogares sustentables, pandemia de COVID 19 y procesos educativos participativos, los cuales se conceptualizan a continuación:

La Organización de las Naciones Unidas para la Alimentación y la Agricultura (FAO) define la Seguridad Alimentaria (SA) como la situación que existe cuando todas las personas tienen, en todo momento, acceso físico, social y económico a alimentos suficientes, inocuos y nutritivos que satisfacen sus necesidades nutrimentales diarias y preferencias alimentarias para llevar una vida activa y sana. Los cuatro dominios que comprenden la SA son: disponibilidad física, acceso económico y físico a los alimentos, la utilización y la estabilidad en el tiempo de las tres dimensiones anteriores, y están descritas en la enciclopedia de SA y sostenibilidad (FAO, 1996.)

La Inseguridad Alimentaria (IA) se define como la disponibilidad limitada o incierta de acceso a alimentos adecuados y culturalmente apropiados por falta de dinero u otros recursos (FAO, FIDA y PMA. 2012.)

Con base en los conceptos de Dietas Sostenibles (Mason, Lang, 2017) y Modos de Vida Sustentables (Pat Fernández, 2012) Crocker y cols. (2019) desarrollan el concepto de hogares sustentables como una propuesta teórico-metodológica para analizar la vulnerabilidad alimentaria de las familias, incluyendo aspectos económicos, sociales, culturales y ambientales, al vincular el concepto de Nutrición Ecológica con el consumo alimentario de las poblaciones y la transformación del modo de producción de alimentos con el objetivo de detener el cambio climático del planeta.

La pandemia de COVID-19 es una crisis sanitaria mundial causada por un coronavirus SARS-CoV 2, recién descubierto. (Di Gennaro, 2020) COVID-19, es mucho más que una enfermedad infecciosa; está afectando la seguridad socioeconómica y alimentaria en todo el mundo. El impacto del virus en SA no está claro, el Marco de las Naciones Unidas para la respuesta socioeconómica inmediata informó que el virus probablemente aumentaría la pobreza, la inseguridad alimentaria y las desigualdades a escala mundial (Pérez-Escamilla, 2017; United Nations, 2020).

La educación participativa es una propuesta fundamentada en la Pedagogía Crítica de Frontera de Henry Giroux (González, 2007.) en la cual los alumnos cogestionan su proceso de aprendizaje con las comunidades y los profesores para generar propuestas de transformación social. "En ese sentido, el conocimiento, las destrezas y los valores se convierten en contenidos educativos necesarios para que el alumno pueda negociar de manera crítica los límites culturales que le ofrece la sociedad y, en 


\section{Artículo Original}

consecuencia, para proceder a transformar el mundo en que vive..."

La presente investigación pretende innovar el conocimiento en la relación seguridad alimentaria familiar y el desarrollo de hogares sustentables de alumnos en instituciones educativas que forman profesionales de la Nutrición, que permitan construir una nueva cultura de alimentación sostenible en las escuelas y facultades de Nutrición y se avance en el desarrollo de universidades sustentables desde la construcción socio-familiar.

\section{Material y Método}

El estudio se fundamenta en una propuesta epistemológica y epidemiológica Crítica, en donde se retoman los aportes de la Teoría Crítica relacionados con el espacio y la geografía urbana valorados desde la sustentabilidad territorial en una perspectiva de la determinación social de la salud (Breihl, 2010). Un aspecto distintivo de esta perspectiva, es que el investigador y los alumnos investigados, establecen una relación de igualdad para interpretar los datos y construir conjuntamente propuestas y acciones de transformación al problema investigado en sus núcleos familiares. En la investigación, el investigador y los investigadores internos, alumnos practicantes que rotan en el Subprograma de Talentos Jóvenes del Programa de Educación, Salud, Alimentación y Ambiente en Comunidades (PROESANC) del Instituto Regional de Investigación en Salud Pública (IRISP) de la Universidad de Guadalajara, conducen un proceso educativo co-gestivo, establecido a través de medios virtuales de aprendizaje, dada la situación de confinamiento de los alumnos practicantes y sus familias, para realizar un diagnóstico situacional de la seguridad alimentaria de los alumnos practicantes y con base en el análisis de datos, establecen un programa operativo durante el semestre para generar acciones de transformación en dirección de propiciar hogares sustentables.

El universo del estudio está constituido por 122 familias de alumnos practicantes de $6^{\circ}$ y $8^{\circ}$ Semestres de la Carrera de Nutrición que realizan sus prácticas profesionales en el otoño del 2020, en donde seleccionan a 67 familias que corresponden a $55 \%$ del total. Las familias participantes corresponden al $100 \%$ de los alumnos del turno matutino; el principal criterio de inclusión es que fueran practicantes de la
Carrera de Nutrición y que aceptaran participar en el estudio.

Se aplica un diseño metodológico de Investigación Acción Participativa (IAP) (Colmenares, 2012.) que incluye tres fases, educación y capacitación inicial, investigación de campo y acciones de transformación. Durante el proceso se aplican técnicas cuantitativas, a través de aplicación de una encuesta estandarizada y la construcción de bases de datos en Excel y Epi Info, así como, técnicas de análisis del discurso escrito para interpretar las estrategias, acciones y recursos utilizados por los alumnos y sus familias para formular un plan operativo de hogar sustentable.

La investigación se realiza en tres fases en donde se implementan los siguientes procedimientos de investigación: En la primera, los alumnos que realizan prácticas profesionales iniciales y terminales en la Orientación Especializante Selectiva de Alimentación y Nutrición en Poblaciones, son capacitados en el uso de una encuesta estandarizada semi-abierta, construida en PROESANC con base en elementos de la cadena epidemiológica de la seguridad alimentaria formulados por el Instituto de Nutrición de Centro América y Panamá (INCAP, 1998) que contiene indicadores de producción, disponibilidad, consumo, aprovechamiento de alimentos y evaluación del estado nutricio individual, familiar, su relación con comorbilidades crónico-degenerativas y la prevalencia de COVID 19 en las familias; asimismo, los alumnos practicantes adquieren competencias para realizar gestión social familiar para desarrollar propuestas cualitativas de hogares sustentables. Esta actividad se realiza en grupos pequeños en el Laboratorio de Agroecología para la Salud, que es un espacio abierto, en donde participan 16 alumnos de $8^{\circ}$. Ciclo, que capacitan en línea a 37 alumnos de $6^{\circ}$ ciclo.

En la segunda fase, de recolección de datos cuantitativos, los 67 alumnos practicantes recolectan datos a través de aplicar la encuesta semiestructurada a los miembros de sus familias mediante entrevista oral directa y llenan los datos en la encuesta. Las encuestas de los alumnos de $6^{\circ}$ ciclo y las propias de los alumnos de $8^{\circ}$ ciclo, son enviadas en línea al equipo de investigadores, quienes realizan el vaciado en una base de datos de Excel. Las encuestas son colectadas por los alumnos investigadores y el 
investigador principal, quienes realizan el análisis de los datos utilizando porcentajes para los datos descriptivos y X2 y $\mathrm{P}$ para los datos inferenciales con base en los patrones de referencia de OMS. Para la evaluación del estado nutricio se aplica la metodología de la Encuesta Nacional de Salud y Nutrición (ENSANUT), se utilizaron los puntos de corte de IMC para adultos e IMC/edad, establecidos por la Organización Mundial de la Salud (OMS); además, se ingresaron los datos de niños $<5$ años en el programa WHO ANTHRO, para obtener la puntuación $\mathrm{Z}$ de talla/edad, peso/edad y peso/talla para poder clasificar a aquellos con retraso de crecimiento, insuficiencia ponderal o con emaciación, sobrepeso u obesidad, respectivamente (WHO, 1995; Cuevas, Shamah, Hernández, González, Gómez, Ávital, et al., 2018) y los indicadores propuestos por el Instituto de Nutrición de Centro América y Panamá (INCAP, 2000.)

Los datos de morbilidades asociadas de los miembros de la familia, se documentaron por los alumnos practicantes, a través de interrogatorio de los miembros por medio de recordatorio de los últimos tres meses con base en los informes médicos de las familias.

En la tercera fase, de acciones de transformación, los alumnos de $8^{\circ}$ y $6^{\circ}$ Semestre con asesoría de sus profesores tutores, elaboran un plan operativo semestral, a partir del diagnóstico de la situación de seguridad alimentaria familiar, las comorbilidades detectadas y del grado de sustentabilidad alimentaria familiar. En esta fase, el equipo de investigación aplica la técnica de análisis de discurso documental para evaluar las estrategias, acciones y recursos utilizados en cada familia de los alumnos para construir la propuesta de hogares sustentables.

La presente investigación se lleva a cabo con los códigos de investigación estipulados por la Declaración de Helsinki y Los Principios Éticos y el Código de Conducta de la American Phychological Association, donde se establece que todos los participantes: deben estar informados del propósito de la investigación y el uso que se hará con los resultados de la misma; se realiza con respeto por la dignidad humana, la igualdad, la autonomía individual y la libertad de expresión, así como la justicia y el acceso a la información.

\section{Resultados}

Se evalúa a 67 familias de alumnos practicantes $(54.91 \%)$ de un universo de 122 familias de alumnos. Las familias de los alumnos están constituidas por 151 mujeres $(56.55 \%)$ y 116 hombres (43.44\%). El 26.59\% $\quad(n=71)$ de los individuos tiene entre 1 y 20 años, $34.08 \%(n=91)$ entre 21 y 35 años y el $39.32 \%(n=105)$ es > 36 años. $\mathrm{El}$ ingreso familiar mensual promedio corresponde a 161.48 salarios mínimos (\$19,897.02 moneda nacional), del cual destinan en promedio a alimentos lo equivalente a 49.31 salarios mínimos $(\$ 6,076$ moneda nacional) que equivale al $30.5 \%$ de sus ingresos mensuales.

En el estudio se analizan las categorías de la cadena epidemiológica de la SA formulados por INCAP (2000) Al realizar la valoración de la categoría producción de alimentos, el $55 \%$ de las familias cuentan con espacio para producir alimentos, sin embargo, sólo el $19.40 \%(\mathrm{n}=13)$ de las familias producen alimentos y/o plantas medicinales en el hogar. En cuanto al tipo de producción predominan el cultivo de frutas $(61.54 \%)$, plantas medicinales $(53.85 \%)$ y verduras $(46.15 \%)$. En ningún hogar se producen legumbres.

Esto coincide con un problema que plantean los alumnos practicantes relacionado con la falta de conocimiento en producción de alimentos para el autoconsumo. En el plan operativo, elaborado por los practicantes para resolver esta problemática, se incluye como acción prioritaria realizar sesiones educativas y prácticas para la promoción de agroecología y huertos familiares en casa, para la creación de una cultura de hogar sustentable, como se menciona en el relato siguiente:

"No solamente asegura (sic) nuestra seguridad alimentaria teniendo acceso y disponibilidad alimentos sanos para nuestra alimentación, sino que también mejora nuestra salud al consumir alimentos frescos y sin pesticidas o algún otro químico con el que normalmente son rociados los cultivos ..."

En lo que refiere a la categoría disponibilidad de alimentos, el $98.51 \%$ de las familias mencionan que adquieren sus alimentos frescos, el $67.16 \%$ enlatados, el $40.30 \%$ congelados y el $31.34 \%$ adquiere sus alimentos preparados. Así mismo, las familias participantes mencionan que utilizan más de un método de almacenaje para sus alimentos, todas 
almacenan sus alimentos por medio de refrigeración, mientras que el $41 \%$ también almacena a la intemperie, el $8.96 \%$ en conservas y el $2.99 \%$ por secado. En la figura 1, se muestran las fuentes de obtención de alimentos, entre las que se destacan la tienda, el mercado y el supermercado con una frecuencia mayor al 70\% y solamente el $10.4 \%$ los obtienen de producción en casa.

Figura 1. Fuentes de obtención de alimentos

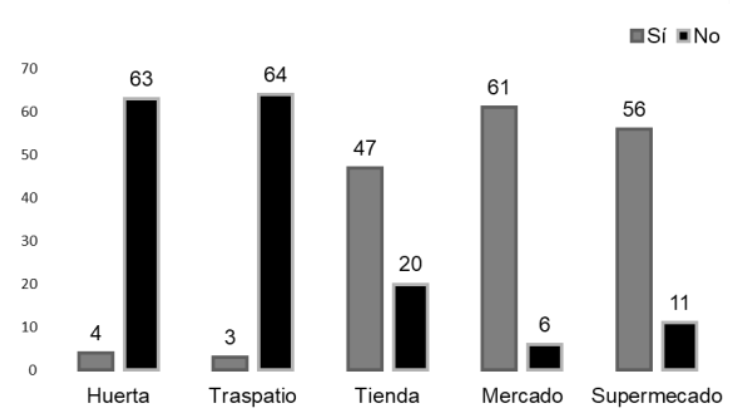

Fuente: Entrevistas a alumnos practicantes

Respecto a la categoría consumo de alimentos, al analizarla con el indicador Frecuencia de Consumo de Alimentos se reporta que todos los miembros de las familias consumen cereales a diario, aceites y grasas en el $94 \%$ de los participantes. Entre los alimentos más consumidos se encuentran las frutas y verduras, consumidas a diario por el $74 \%$ de los miembros de las familias. También se reporta un consumo frecuente de lácteos, alimentos cárnicos y azúcares, consumidos a diario por el $53.73 \%, 52.23 \%$ y $49.25 \%$ respectivamente.

En la categoría aprovechamiento de alimentos, el $100 \%$ de las familias cuentan con los servicios básicos de agua, luz y drenaje; $98.51 \%$ con gas y $97 \%$ con recolección de basura. Únicamente 34 $(50.75 \%)$ de las familias realizan separación de residuos, y de estas sólo el $38 \%$ da un adecuado manejo a los residuos, al reciclarlos o elaborar composta.

El $91.84 \%$ de los alumnos practicantes considera importante en el plan operativo el aprovechamiento de los alimentos como propuesta de intervención, en donde señalan que se impartieron talleres de lombricomposta y composta, separación de basura y su correcta manipulación, y se buscó disminuir el desperdicio de comida, principalmente en los residuos, uno de ellos señala lo siguiente:

"Aproximadamente se genera de 2 a 3 kilos de basura al día por familia, de igual manera y generalmente se puede decir que estos hogares no realizan acciones como el separar la basura, ni reutilizar o reciclar, tampoco tienen un buen ahorro de agua, ni de luz...”

En la categoría estado nutricio y morbilidad asociada se encuentra lo siguiente: la prevalencia combinada de sobrepeso y obesidad de todos los grupos de edad, de acuerdo con el Índice de Masa Corporal (IMC), es de $46.25 \%$, mientras que el $48.87 \%$ se encuentra en normopeso y el $4.89 \%$ en bajo peso. El $37 \%$ de los niños $<5$ años $(\mathrm{n}=8)$, tiene retraso de crecimiento, valorado por el indicador talla/edad, $25 \%$ tiene insuficiencia ponderal (indicador peso/edad) y también un $25 \%$ tiene emaciación leve (indicador peso/talla); mientras que el $37.5 \%$ se encuentra por arriba del estándar de normalidad de peso para la talla. Se observa una diferencia significativa en la relación de IMC y sexo ( $\mathrm{p}=0.03)$, así como para IMC $\mathrm{y}$ edad $(\mathrm{p}=0)($ tablas 1 y 2$)$.

Tabla 1. Relación Índice de Masa Corporal y Covid-19

\begin{tabular}{lrrrrrr}
\hline Clasificación & \multicolumn{2}{r}{ Femenino } & \multicolumn{2}{c}{ Masculino } & \multicolumn{2}{c}{ Total } \\
\multicolumn{1}{c}{ IMC } & $\mathrm{n}$ & $\%$ & $\mathrm{n}$ & $\%$ & $\mathrm{n}$ & $\%$ \\
\hline Bajo peso & 5 & 38.5 & 8 & 61.5 & 13 & 4.9 \\
Normopeso & 85 & 65.4 & 45 & 34.6 & 130 & 48.7 \\
Sobrepeso & 40 & 52.6 & 36 & 47.4 & 76 & 28.5 \\
Obesidad & 22 & 45.8 & 27 & 56.3 & 48 & 18.0 \\
Total & 152 & 56.9 & 116 & 43.4 & 267 & 100.0 \\
\hline x $^{2}$ 8.73; $p$ 0.03 & & & & & & \\
Fuente: Entrevistas a practicantes & & & &
\end{tabular}

\begin{tabular}{|c|c|c|c|c|c|c|c|c|c|c|c|c|c|c|}
\hline \multirow{2}{*}{$\begin{array}{c}\text { Clasificación } \\
\text { IMC }\end{array}$} & \multirow{2}{*}{$\begin{array}{l}1-2 \\
n \% \\
\end{array}$} & \multicolumn{2}{|c|}{$3-6$} & \multicolumn{2}{|c|}{$7-12$} & \multicolumn{2}{|c|}{$13-20$} & \multicolumn{2}{|c|}{$21-35$} & \multicolumn{2}{|c|}{$36-60$} & \multirow{2}{*}{$\frac{>60}{n}$} & \multicolumn{2}{|c|}{ Total } \\
\hline & & $n$ & $\%$ & $n^{-}$ & $\%$ & $n$ & $\%$ & $n$ & $\%$ & $\mathrm{n}$ & $\%$ & & $n$ & $\%$ \\
\hline Bajo peso & $\begin{array}{lll}1 & 7.7\end{array}$ & & 15.4 & 0 & 0.0 & 5 & 38.5 & 3 & 23.1 & 2 & 15.4 & $\begin{array}{ll}0 & 0.0\end{array}$ & 13 & 4.9 \\
\hline Normopeso & $\begin{array}{ll}1 & 0.8\end{array}$ & 6 & 4.6 & 10 & 7.7 & 26 & 20.0 & 58 & 44.6 & 25 & 19.2 & $\begin{array}{lll}4 & 3.1\end{array}$ & 130 & 48.7 \\
\hline Sobrepeso & $\begin{array}{ll}0 & 0.0\end{array}$ & 1 & 1.3 & 0 & 0.0 & 3 & 3.9 & 19 & 25.0 & 51 & 67.1 & 22.6 & 76 & 28.5 \\
\hline Obesidad & $\begin{array}{ll}1 & 2.1\end{array}$ & 1 & 2.1 & & 16.7 & 6 & 12.5 & 11 & 22.9 & 18 & 37.5 & $\begin{array}{l}36.3 \\
\end{array}$ & 48 & 18.0 \\
\hline Total & $\begin{array}{ll}3 & 1.1\end{array}$ & 10 & 3.7 & 18 & 6.7 & 40 & 15.0 & 91 & 34.1 & 95 & 35.6 & $\begin{array}{ll}9 & 3.4 \\
\end{array}$ & 267 & 100.0 \\
\hline
\end{tabular}

En los datos de morbilidad general, el $6 \%$ de la muestra ha estado enferma por Covid-19. El 10.5\% presenta alguna enfermedad previa, de las cuales el $28.57 \%$ son enfermedades gastrointestinales (GI), el $21.43 \%$ enfermedades cardiovasculares y $17.86 \%$ 
enfermedades endócrinas. Al realizar un análisis por grupos de edad, predominan en un $28.57 \%$ las enfermedades GI en niños y adultos jóvenes (11-35 años), mientras que las enfermedades cardiovasculares, endocrinas y renales, en adultos de 36-60 años (42.85\%). La relación entre comorbilidad previa y presencia de Covid-19 (tablas 3 y 4) muestran una asociación positiva entre las variables, sin embargo, el sobrepeso/obesidad relacionado a la presencia de Covid-19 (tabla 5) no muestra resultados significativos, probablemente por ser adultos con menor edad.

Tabla 3. Cormobilidad y Covid-19

\begin{tabular}{|c|c|c|c|c|c|c|}
\hline & \multicolumn{4}{|c|}{ Presencia COVID-19 } & \multirow{2}{*}{\multicolumn{2}{|c|}{ Total }} \\
\hline & \multicolumn{2}{|c|}{$\mathrm{SI}$} & \multicolumn{2}{|c|}{ No } & & \\
\hline & $\mathrm{n}$ & $\%$ & $\mathrm{n}$ & $\%$ & $\mathrm{n}$ & $\%$ \\
\hline \multicolumn{7}{|c|}{ Comorbilidad previa* } \\
\hline $\mathrm{Si}$ & 4 & 14.3 & 24 & 85.7 & 28 & 10.5 \\
\hline No & 12 & 5.0 & 227 & 95.0 & 239 & 89.5 \\
\hline \multicolumn{7}{|c|}{ Tipo de comorbilidad** } \\
\hline Cardiovascular & 1 & 16.7 & 5 & 83.3 & 6 & 21.4 \\
\hline Endocrina & 0 & 0.0 & 5 & 100.0 & 5 & 17.9 \\
\hline Gastrointestinal & 3 & 42.9 & 4 & 57.1 & 7 & 25.0 \\
\hline Respiratoria & 0 & 0.0 & 3 & 100.0 & 3 & 10.7 \\
\hline Autoinmune & 0 & 0.0 & 2 & 100.0 & 2 & 7.1 \\
\hline De la piel & 0 & 0.0 & 23 & 1150.0 & 2 & 7.1 \\
\hline De la sangre & 0 & 0.0 & 1 & 100.0 & 1 & 3.6 \\
\hline Renal & 0 & 0.0 & 2 & 100.0 & 2 & 7.1 \\
\hline
\end{tabular}

Tabla 4. Relación sobrepeso/obesidad y Covid-19

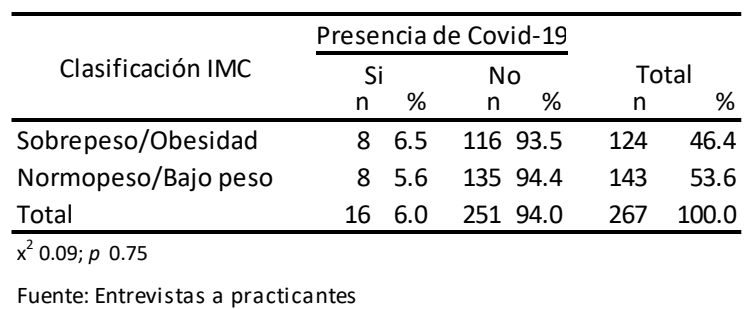

Las comorbilidades relacionadas a la edad no presentan diferencias significativas $(\mathrm{p}=0.17)$ al igual que la relación de comorbilidad para edad y sexo femenino $(\mathrm{p}=0.27)$ sin embargo, sí presentó una asociación positiva en el sexo masculino $(\mathrm{p}=0.03)$.
El sobrepeso y obesidad relacionada a enfermedades crónico degenerativas no presenta resultados significativos $(\mathrm{p}=0.57)$, al igual que el estado nutricio relacionado a la producción de alimentos $(\mathrm{p}=0.47)$.

En la categoría estilo de vida: los niños y adolescentes de 1-20 años, realizan actividad física (AF) en promedio 2.4 días/semana $\pm 2.1 \mathrm{DE}$ y consumen agua $(\mathrm{CA}) 1.3 \mathrm{Lt} /$ día de agua natural \pm 0.8 DE; la población joven adulta entre 21 y 35 años realiza AF en promedio 3.2 días/semana $\pm 2.2 \mathrm{DE}$ y consumen $2 \mathrm{Lt} /$ día de agua $\pm 0.8 \mathrm{DE}$, mientras que los adultos >36 años realizan 1.8 días/semana de AF $\pm 2.2 \mathrm{DE}$ y consumen $1.5 \mathrm{Lt} /$ día de agua $\pm 0.6 \mathrm{DE}$ en promedio.

En las propuestas de plan operativo de los alumnos para un estilo de vida saludable y sustentable, se promueve la promoción de la importancia de pausas activas, AF de acuerdo con las capacidades del adulto mayor, recomendaciones de AF de la Organización Mundial de la salud (OMS) y actividades recreativas en casa para toda la familia, esto en palabras de los practicantes:

"Para que los niños puedan crear el hábito de realizar actividad física con el paso del tiempo [se debe] concientizar sobre las ventajas de realizarla para prevenir complicaciones de sobrepeso $y$ obesidad en este grupo etario..."

\section{Discusión}

Con base en los datos sociodemográficos y de saneamiento básico, las familias de los alumnos practicantes estudiadas están constituidas por población de capas medias, con capacidad de compra de alimentos y con buenos servicios básicos, en donde predomina la población joven y adulta.

De acuerdo a la categoría de producción de alimentos, los hogares de las familias de los alumnos practicantes no tienen una práctica de sustentabilidad, a pesar de que más de la mitad de las familias tienen espacios para cultivo, agua intubada para producirlos y desechos orgánicos para elaborar compostas u otros abonos orgánicos, sin embargo, los alumnos tienen conciencia del problema y lo incluyen como una prioridad en sus planes operativos.

Con respecto a los datos de las categorías de disponibilidad y consumo de alumnos, la mitad de las 
familias tienen dietas de baja calidad biológica y nutricional debido al consumo distorsionado de alimentos, lo que repercute en que la mitad de los miembros de las familias de los alumnos practicantes tengan sobrepeso y obesidad, con impacto en la prevalencia de enfermedades crónico-degenerativas, principalmente enfermedades cardiovasculares, como comorbilidades para padecer COVID 19, a pesar de que no se encontró asociación estadística con sobrepeso y obesidad.

En el análisis de los datos estilos de vida saludables valorados por actividad física y consumo de agua, la primera (AF) es baja y la segunda (CA) es normal, de acuerdo a las recomendaciones internacionales de OMS.

La forma en que se valora la seguridad alimentaria en México se relaciona únicamente con indicadores de disponibilidad y consumo de alimentos, por lo que no es posible contrastar los resultados del estudio con las estadísticas que se formulan en el país. En el presente estudio la seguridad alimentaria se valora con las categorías formuladas por el INCAP (2000) que incluyen: producción, disponibilidad, consumo y aprovechamiento de alimentos y su relación con el estado nutricio individual y familiar. Con los resultados empíricos recolectados en la investigación, las familias se encuentran en inseguridad alimentaria en las categorías analizadas. En lo que se refiere a los conceptos de hogares sustentables y modos de vida sostenibles (Mason y Lang, 2017) (Fernández, 2012) (Crocker, 2019) la mayoría de las familias tienen vulnerabilidad alimentaria, principalmente en los aspectos sociales, culturales y ambientales, por lo que se puede inferir que no contribuyen al objetivo de detener el cambio climático en los espacios urbanos donde viven.

De acuerdo a los planes operativos formulados por los alumnos practicantes y sus profesores tutores, que son analizados de forma cualitativa, existe conciencia de la importancia de transitar hacia hogares y modos de vida sustentables, para aportar en la dirección del concepto de Nutrición Ecológica (Lang, 2017) y mejorar los indicadores de seguridad alimentaria, a través de promover dietas sustentables, principalmente en disminuir el consumo de carnes rojas y alimentos ricos en azúcares simples.

Las comorbilidades relacionadas con sobrepeso y obesidad están asociadas con la alta prevalencia de enfermedades crónico-degenerativas, principalmente cardiovasculares y renales, y se encuentran asociadas a la prevalencia de COVID-19, como se expresan en los estudios realizados en España y México.

De acuerdo a la experiencia educativa vivida con los alumnos practicantes y sus familias, durante el confinamiento por COVID-19, es importante desarrollar metodologías participativas para la investigación y el aprendizaje en línea con la perspectiva Crítica (Breihl, 2010) en los programas de alimentación poblacional de las licenciaturas en Nutrición del país, que permita realizar programas de incidencia en la promoción de hogares sustentables y dietas sostenibles en el hogar y los espacios geográficos donde viven los alumnos para impactar el cambio climático urbano.

\section{Conclusiones}

Las familias de los alumnos practicantes en Nutrición tienen inseguridad alimentaria de acuerdo a los indicadores de producción y consumo de alimentos, lo que impacta en los indicadores de estado nutricio, valorados por IMC y las comorbilidades relacionadas con la prevalencia de enfermedad crónico degenerativa asociada a COVID-19.

Las familias de los alumnos practicantes de la Carrera de Nutrición del Centro Universitario de Ciencias de la Salud de la Universidad de Guadalajara viven en hogares no sustentables y no consumen dietas sostenibles, lo que impacta en su vulnerabilidad alimentaria.

Se recomienda desarrollar proyectos que impacten los espacios geográficos urbanos y rurales en las carreras de nutrición, principalmente en los programas de salud pública y alimentación poblacional que permitan desarrollar modos de vida sustentables a escala familiar y poblacional, así como, en los espacios universitarios, en dirección de tomar conciencia del cambio climático planetario. 


\section{Bibliografía}

Bello-Chavolla, O. Y., Bahena-López, J. P., Antonio-Villa, N. E., Vargas-Vázquez, A., González-Díaz, A., Márquez-Salinas, A., Fermín-Martínez, C. A., Naveja, J. J., y AguilarSalinas, C. A. (2020). Predicting Mortality Due to SARS-CoV-2: A Mechanistic Score Relating Obesity and Diabetes to COVID-19 Outcomes in Mexico. The Journal of clinical endocrinology and metabolism, 105(8), dgaa346.

Breihl, J. (2010). La epidemiología crítica: una nueva forma de mirar la salud en el espacio urbano. Rev. Salud Colectiva, Buenos Aires, 6(1):83-101

Colmenares, A. M. (2012) Investigación-acción participativa: una metodología integradora del conocimiento y la acción. Voces y Silencios: Revista Latinoamericana de Educación, Vol. 3, No. 1, 102-115.

Crocker, R., Muñoz P., Vázquez J. L., Pérez T. (2019) Relación del modo de producción de alimentos y vida sustentable en la microcuenca la Primavera-Presa de la Vega en el estado de Jalisco, México. Oikos Polis, Rev. Latinoamericana de Ciencias Económicas y Sociales. 4 (2) 1-29.

Eche, D., \& Hernández-Herrera, M. (2018). Studying food security among students: a comparative case study between public and private universities in Quito-Ecuador. Nutrición Hospitalaria, 35(6), 1372-1378.

Elsahoryi N., Al-Sayyed H., Odeh M., McGrattan A., Hammad F. (2020). Effect of Covid-19 on food security y: A cross-sectional survey. Clinical Nutrition ESPEN. DOI: https://doi.org/10.1016/j.clnesp.2020.09.026

FAO, FIDA y PMA. (2012) El estado de la inseguridad alimentaria en el mundo 2012. El crecimiento económico es necesario, pero no suficiente para acelerar la reducción del hambre y la malnutrición. Roma, FAO. http://www.fao.org/3/i2845s/i2845s00.pdf

Fernández P., Lucio A., Nahed J., Téllez J, y García N. (2012) "Modos de vida sustentables una metodología para el estudio de la seguridad alimentaria. "Revista Salud Pública y Nutrición $13(1)$.

https://respyn.uanl.mx/index.php/respyn/issue/vi ew/68

González L. (2007) La Pedagogía Crítica de Henry A. Giroux. Rev. Sinéctica, 29-86. URL: Pedagogía de Frontera Giroux.pdf

OPS/INCAP. (2000) Hacia la seguridad alimentaria y nutricional en el Siglo XXI. Memorias de Reunión Científica. Guatemala, 68 septiembre de 1999.

Kammar-García, A., Vidal-Mayo, J. J., VeraZertuche, J. M., Lazcano-Hernández, M., VeraLópez, O., Segura-Badilla, O., Aguilar-Alonso, P., y Navarro-Cruz, A. R. (2020). Imparct of comorbidities in mexican SARS-COV-2-positive patients: A retrospective analysis in a national cohort. Revista de Investigación Clínica; Órgano del Hospital de Enfermedades de la Nutrición, 72(3), 151-158.

Mason P., Lang T. (2017) Sustainable diets: how ecological nutrition can transform consumption and the food system Routledge, Oxon, UK.

Mundo-Rosas, V., Vizuet-Vega, N. I., MartínezDomínguez, J., Morales-Ruán, M. C., PérezEscamilla, R., \& Shamah-Levy, T. (2018). Evolución de la inseguridad alimentaria en los hogares mexicanos: 2012-2016. Salud Pública de México, 60(3), 309-318. Obtenido de https://www.medigraphic.com/pdfs/salpub

Organización de las Naciones Unidas para la Agricultura y la Alimentación. 1996. "Informe de políticas, seguridad alimentaria. Cumbre Mundial de la Alimentación en Roma, Italia" Dirección de Economía Agrícola y del Desarrollo.

Posso, M., Comas, M., Román, M., Domingo, L., Louro, J., González, C., Sala, M., Anglès, A., Cirera, I., Cots, F., Frías, V. M., Gea, J., GüerriFernández, R., Masclans, J. R., Noguès, X., Vázquez, O., Villar-García, J., Horcajada, J. P., Pascual, J., y Castells, X. (2020). Comorbidities and Mortality in Patients with COVID-19 Aged 60 Years and Older in a University Hospital in 


\section{RESPYN}

Spain. Archivos de Bronconeumología, 56(11), 756-758.

Wilson, N., Cleghorn, C., Cobiac, L., Mizdrak, A., y Nghiem, N. (2019). Achieving Healthy and Sustainable Diets: A Review of the Results of Recent Mathematical Optimization Studies. Advances in Nutrition, 10, S389-S203. 